\title{
The rank function of a positroid and non-crossing partitions
}

\author{
Robert Mcalmon \\ Department of Mathematics \\ Texas State University \\ Texas, U.S.A. \\ rgmcalmon@txstate.edu
}

\author{
Suho Oh \\ Department of Mathematics \\ Texas State University \\ Texas, U.S.A. \\ s_o79@txstate.edu
}

Submitted: Oct 21, 2018; Accepted: Dec 14, 2019; Published: Jan 10, 2020

(C) Robert Mcalmon and Suho Oh. Released under the CC BY-ND license (International 4.0).

\begin{abstract}
A positroid is a special case of a realizable matroid that arose from the study of totally nonnegative part of the Grassmannian by Postnikov [13]. Postnikov demonstrated that positroids are in bijection with certain interesting classes of combinatorial objects, such as Grassmann necklaces and decorated permutations. The bases of a positroid can be described directly in terms of the Grassmann necklace and decorated permutation [10]. In this paper, we show that the rank of an arbitrary set in a positroid can be computed directly from the associated decorated permutation using non-crossing partitions.
\end{abstract}

Mathematics Subject Classifications: 05A17, 05E99

\section{Introduction}

A totally nonnegative matrix is a square matrix where all of its minors are nonnegative real numbers. These matrices have remarkable properties. For example, the space of these matrices can be grouped into topological cells, with each cell parametrized by a certain planar network [4]. The notion of total positivity has numerous applications and has been studied from many different angles, including oscillations in mechanical systems, stochastic processes and approximation theory, and planar resistor networks [4].

Now, instead of considering $n \times n$ matrices, consider a full-rank $k \times n$ matrix with all maximal minors nonnegative. It turns out these matrices can also be grouped into topological cells inside the Grassmannian, with each cell again being parametrized by a certain planar network [13]. The set of nonzero maximal minors of such matrices forms a positroid, which is a matroid used to encode the cells. Positroids have a number of nice combinatorial properties. In particular, Postnikov demonstrated that positroids are in bijection with certain interesting classes of combinatorial objects, such as Grassmann 
necklaces and decorated permutations. Recently, positroids have seen increased applications in physics, with use in the study of scattering amplitudes [2] and the study of shallow water waves [7].

Cyclic intervals and non-crossing partitions have proven to be extremely useful for describing positroids. A positroid polytope can be compactly described in terms of cyclic intervals by utilizing the Grassmann necklace description for bases of a positroid $[1],[8],[9],[10]$. It was shown in [1] that non-crossing partitions and positroids are intimately related through a positroid's connected components. In this paper, we provide yet another usage of cyclic intervals and non-crossing partitions for positroids.

Given an arbitrary set, its rank (the size of the biggest intersection with a basis) can be obtained by going through all the bases. In this paper, we show a method of obtaining the rank of an arbitrary set directly from the associated decorated permutation without having to go through the bases. In particular, we describe a collection of upper bounds of the rank coming from non-crossing partitions, and one of them will be shown to be tight.

The structure of the paper is as follows. In Section 2, we go over the background materials needed for this paper, including the basics of matroids, positroids, Grassmann necklaces and decorated permutations. In Section 3 we develop a transformation process for Grassmann necklace elements. In Section 4, we show our main result that the rank of an arbitrary set in a positroid can be obtained directly from the decorated permutation by using non-crossing partitions. In Section 5, we provide an example of how to use our main result to compute the rank of a set.

\section{Acknowledgement}

The authors would like to thank Lillian Bu, Wini Taylor-Williams and David Xiang for useful discussions.

\section{Background materials}

\subsection{Matroids}

In this section we review the basics of matroids that we will need. We refer the reader to [12] for a more in-depth introduction to matroid theory.

Definition 1. A matroid is a pair $(E, \mathcal{B})$ consisting of a finite set $E$, called the ground set of the matroid, and a nonempty collection of subsets $\mathcal{B}=\mathcal{B}(\mathcal{M})$ of $E$, called the bases of $\mathcal{M}$, which satisfy the basis exchange axiom:

If $B_{1}, B_{2} \in \mathcal{B}$ and $b_{1} \in B_{1} \backslash B_{2}$, then there exists $b_{2} \in B_{2} \backslash B_{1}$ such that $B_{1} \backslash\left\{b_{1}\right\} \cup\left\{b_{2}\right\} \in \mathcal{B}$.

A subset $F \subseteq E$ is called independent if it is contained in some basis. All maximal independent sets contained in a given set $A \subseteq E$ have the same size, called the rank $\operatorname{rk}(A)$ of $A$. The rank of the matroid $\mathcal{M}$, denoted as $\operatorname{rk}(\mathcal{M})$, is given by $\operatorname{rk}(E)$. An element $e \in E$ is a loop if it is not contained in any basis. An element $e \in E$ is a coloop if it is contained in all bases. A matroid $\mathcal{M}$ is loopless if it does not contain any loops. 
The $\boldsymbol{d u a l}$ of $\mathcal{M}$ is a matroid $\mathcal{M}^{*}=\left(E, \mathcal{B}^{\prime}\right)$ where $\mathcal{B}^{\prime}=\{E \backslash B \mid B \in \mathcal{B}(\mathcal{M})\}$. By using the basis exchange axiom on the dual matroid, we get the following dual basis exchange axiom:

If $B_{1}, B_{2} \in \mathcal{B}$ and $b_{2} \in B_{2} \backslash B_{1}$, then there exists $b_{1} \in B_{1} \backslash B_{2}$ such that $B_{1} \backslash\left\{b_{1}\right\} \cup\left\{b_{2}\right\} \in \mathcal{B}$.

Remark 2. In this paper, we will always use $[n]:=\{1, \ldots, n\}$ as our ground set, reserving the usage of $E$ for subsets of the ground set we analyze. A matroid of rank $d$ will have bases in the set $\left(\begin{array}{c}{[n]} \\ d\end{array}\right)$ which stands for all cardinality $d$-subsets of $[n]$. Also for ease of reading we will use $B \in \mathcal{M}$ to denote $B \in \mathcal{B}(\mathcal{M})$.

Let $E$ be an arbitrary subset of the ground set $[n]$. For a basis $J$, if $|J \cap E|$ is maximal among $|B \cap E|$ for all bases $B$ of the matroid $\mathcal{M}$, we say that $J$ maximizes $E$, or $J$ is maximal in $E$. Similarly, if $|J \cap E|$ is minimal among $|B \cap E|$ for all bases $B$ of $\mathcal{M}$, we say that $J$ minimizes $E$, or $J$ is minimal in $E$.

The following property of the rank function will be crucial:

Theorem 3 ([12]). The rank function is semimodular, meaning $\operatorname{rk}(A \cup B)+\operatorname{rk}(A \cap B) \leqslant$ $\operatorname{rk}(A)+\operatorname{rk}(B)$ for any subset $A$ and $B$ of the ground set.

Consider a matrix with entries in $\mathbb{R}$ that has $n$ columns and $r$ rows, with $r \leqslant n$. Column sets that correspond to $r$-by- $r$ submatrices with nonzero determinant generate (the set of bases of) a matroid. Such matroids are called realizable matroids. For example, consider the following matrix:

$$
A=\left(\begin{array}{cccc}
1 & 0 & -3 & -1 \\
0 & 1 & 4 & 0
\end{array}\right)
$$

The column sets $\{1,2\},\{1,3\},\{2,3\},\{2,4\},\{3,4\}$ correspond to 2 -by-2 submatrices that have nonzero determinant. So, the collection $\{\{1,2\},\{1,3\},\{2,3\},\{2,4\},\{3,4\}\}$ over the ground set [4] is a realizable matroid.

Proposition 4. Let $\mathcal{M}$ be a realizable matroid over the ground set $[n]$, and let $B$ be a basis of $\mathcal{M}$. Pick an arbitrary subset $E$ of $[n]$ such that $B$ maximizes $E$ and there is some $J \subseteq E$ such that $|J|=\operatorname{rk}(J)=\operatorname{rk}(E)$. Then $B \backslash(B \cap E) \cup J$ is another basis of $\mathcal{M}$.

Proof. By the assumption $|J|=\operatorname{rk}(J)=\operatorname{rk}(E)$, it follows $\operatorname{span}(E)=\operatorname{span}(J)$ where $\operatorname{span}(E)$ denotes the span of the column vectors corresponding to $E$. Moreover, since $B$ maximizes $E$, then $\operatorname{span}(B \cap E)=\operatorname{span}(J)$. Notice $|J|=\operatorname{rk}(J)$ implies $J \subset B^{\prime}$ for some basis $B^{\prime}$. Then by the basis exchange axiom, $B \backslash(B \cap E) \cup J$ is a basis of $\mathcal{M}$.

\subsection{Positroids}

In this section we go over the basics of positroids. Positroids were originally defined in [13] as the column sets coming from nonzero maximal minors of a matrix such that all maximal minors are nonnegative. For example, the realizable matroid generated by matrix $A$ in the previous section is in fact a positroid since its column sets correspond to nonzero maximal minors of $A$. However in this paper, we will use an equivalent definition using Grassmann necklaces and Gale orderings. 
Definition 5. Let $d \leqslant n$ be positive integers. A Grassmann necklace of type $(d, n)$ is a sequence $\left(I_{1}, \ldots, I_{n}\right)$ of $d$-subsets $I_{k} \in\left(\begin{array}{c}{[n]} \\ d\end{array}\right)$ such that for any $i \in[n]$,

- if $i \in I_{i}$ then $I_{i+1}=I_{i} \backslash\{i\} \cup\{j\}$ for some $j \in[n]$,

- if $i \notin I_{i}$ then $I_{i+1}=I_{i}$,

where $I_{n+1}=I_{1}$.

The cyclically shifted order $<_{i}$ on the set $[n]$ is the total order

$$
i<_{i} i+1<_{i} \cdots<_{i} n<_{i} 1<_{i} \cdots<_{i} i-1 .
$$

For any rank $d$ matroid $\mathcal{M}$ with ground set $[n]$, let $I_{k}$ be the lexicographically minimal basis of $\mathcal{M}$ with respect to $<_{k}$, and denote

$$
\mathcal{I}(\mathcal{M}):=\left(I_{1}, \ldots, I_{n}\right)
$$

which forms a Grassmann necklace [13].

The Gale order on $\left(\begin{array}{c}{[n]} \\ d\end{array}\right)$ (with respect to $<_{i}$ ) is the partial order $<_{i}$ defined as follows: for any two $d$-subsets $S=\left\{s_{1}<_{i} \cdots<_{i} s_{d}\right\}$ and $T=\left\{t_{1}<_{i} \cdots<_{i} t_{d}\right\}$ of $[n]$, we have $S \leqslant_{i} T$ if and only if $s_{j} \leqslant_{i} t_{j}$ for all $j \in[d]$ [5].

Theorem $6([10],[13])$. Let $\mathcal{I}=\left(I_{1}, \ldots, I_{n}\right)$ be a Grassmann necklace of type $(d, n)$. Then the collection

$$
\mathcal{B}(\mathcal{I}):=\left\{B \in\left(\begin{array}{c}
{[n]} \\
d
\end{array}\right) \mid B \geqslant_{j} I_{j}, \text { for all } j \in[n]\right\}
$$

is the collection of bases of a rank $d$ positroid $\mathcal{M}(\mathcal{I}):=([n], \mathcal{B}(\mathcal{I}))$. Here $\geqslant_{j}$ is the aforementioned Gale order. Moreover, for any positroid $\mathcal{M}$, we have $\mathcal{M}(\mathcal{I}(\mathcal{M}))=\mathcal{M}$.

In order to check if a set is a basis of a positroid or not, we do not have to check for all the cyclic orderings.

Corollary 7. Let $\mathcal{M} \subseteq\left(\begin{array}{c}{[n]} \\ d\end{array}\right)$ be a positroid and $\mathcal{I}$ the associated Grassmann necklace. A set $B \in\left(\begin{array}{c}{[n]} \\ d\end{array}\right)$ is a basis of $\mathcal{M}$ if and only if $B \geqslant_{b} I_{b}$ for all $b \in B$.

Proof. For arbitrary $q \in[n]$, denote the elements of $B$ as $b_{1}<_{q} b_{2}<_{q} \cdots<_{q} b_{d}$. If we had $B \geqslant_{b_{1}} I_{b_{1}}$, we would also have $B \geqslant_{q} I_{b_{1}} \geqslant_{q} I_{q}$.

Definition 8. A decorated permutation of the set $[n]$ is a bijection $\pi$ of $[n]$ whose fixed points are colored either white or black. A weak $\boldsymbol{i}$-exceedance of a decorated permutation $\pi$ is an element $j \in[n]$ such that either $j<_{i} \pi^{-1}(j)$ or $j$ is a fixed point colored black.

Given a decorated permutation $\pi$ of $[n]$ we can construct a Grassmann necklace $\mathcal{I}=$ $\left(I_{1}, \ldots, I_{n}\right)$ by letting $I_{k}$ be the set of weak $k$-exceedances of $\pi$. We will envision a decorated permutation by drawing a circle having boundary entries $[n]$ and arrows going from $i$ to $\pi(i)$ for each $i$. To get the $k$-exceedance, cut the circle off between $k-1$ and $k$ 


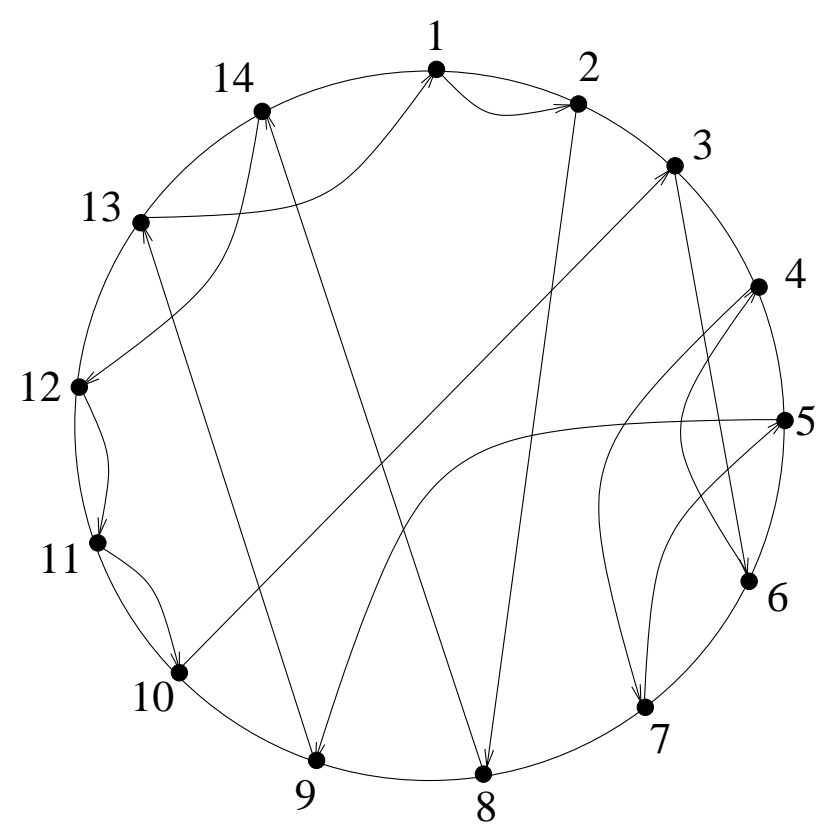

Figure 1: A decorated permutation.

to get a horizontal straight line with leftmost endpoint being $k$ and rightmost endpoint being $k-1$. Redraw the arrows of the permutation accordingly so that it stays within the line. Endpoints of the leftward arrows are exactly the weak $k$-exceedances of $\pi$, hence the elements of $I_{k}$. There is a bijection between Grassmann necklaces and decorated permutations [13].

Example 9. Consider the permutation $[2,8,6,7,9,4,5,14,13,3,10,11,1,12]$ under the usual one-line notation. Since it has no fixed points, this is a decorated permutation. We can draw the permutation by placing the entries of $[n]$ on a circle in clockwise order as in Figure 1. To get $I_{1}$ of the Grassmann necklace of the corresponding positroid, we need to look at the weak 1-exceedances. To do this, cut the circle off between 1 and 14, and straighten the boundary so that 1 to 14 lies on a straight line from left to right. The endpoints of leftward arrows are $\{1,3,4,5,10,11,12\}$ and this is our $I_{1}$.

Remark 10. When we are dealing with positroids, we will always envision the ground set $[n]$ to be drawn on a circle. We will say that $a_{1}, \ldots, a_{t} \in[n]$ are cyclically ordered if there exists some $i \in[n]$ such that $a_{1}<_{i} \cdots<_{i} a_{t}$.

Given $a, b \in[n]$, we define the cyclic interval $[a, b]$ to be the set $\left\{x \mid x \leqslant_{a} b\right\}$. These cyclic intervals play an important role in the structure of a positroid [1],[6],[9]. All intervals mentioned in this paper will actually be referring to cyclic intervals.

Remark 11. It is enough to consider the positroid $\mathcal{M}^{\prime}$ obtained by deleting any loops and coloops in order to study the structural properties of $\mathcal{M}$. For the remainder of this paper, we will assume positroids are loopless and coloopless. This means that the associated decorated permutations have no fixed points. 


\section{$3 \quad$ Intervals and Morphing}

In this section we develop a technique for transforming Grassmann necklace elements. Throughout the paper, unless otherwise stated, we will always be working with a positroid $\mathcal{M}$ on a ground set $[n]$, with rank $d$, having Grassmann necklace $\mathcal{I}=\left(I_{1}, \ldots, I_{n}\right)$, and an associated decorated permutation $\pi$ that does not have any fixed points (see Remark 11). The example positroid that we will be using, again unless otherwise stated, will be the positroid associated to the decorated permutation of Figure 1.

The next property, whose proof is omitted, follows from the definition of a Grassmann necklace.

Lemma 12 (Sharing property). Let $a$ and $b$ be arbitrary elements of $[n]$. Then we have $I_{a} \cap[b, a) \subseteq I_{b} \cap[b, a)$.

Example 13. To illustrate using our running example, notice that since $I_{3}=\{3,4,5,8$, $10,11,12\}$, the set $I_{3} \cap[9,3)=\{10,11,12\}$ is contained in $I_{9}=\{9,10,11,12,14,4,5\}$.

We begin our analysis of the cyclic intervals of a positroid. The following lemma follows directly from Theorem 6 .

Lemma 14. For any interval $[a, b] \subseteq[n]$, the interval is maximized by $I_{a}$. Any interval $(b, a)$ is minimized by $I_{a}$.

This can easily be seen by taking some Grassmann necklace element and any arbitrary basis; say, $I_{6}=\{6,7,8,9,10,11,12\}$ and $B=\{6,7,10,11,12,1,4\}$. Examine how the lemma holds on the intervals $[6,10]$ and $(10,6)$ in $[14]: I_{6} \cap[6,10]=\{6,7,8,9,10\}$ contains more elements than $B \cap[6,10]=\{6,7,10\}$, while $I_{6} \cap(10,6)=\{11,12\}$ contains fewer elements than $B \cap(10,6)=\{11,12,1,4\}$. The above lemma also suggests that given a cyclic interval $[a, b]$, the set $I_{a} \cap[a, b]$ plays a crucial role in studying that interval.

Our goal of the paper is to express the rank of an arbitrary set $E \subseteq[n]$ using noncrossing partitions. To do so, we need to construct the bases that maximize $E$ and analyze them.

Remark 15 . When $E$ is a subset of the ground set $[n]$ and we want to write $E$ as a disjoint union of cyclic intervals so that $E=\left[a_{1}, b_{1}\right] \cup \cdots \cup\left[a_{s}, b_{s}\right]$, we will arrange the $a_{i}$ 's such that $a_{1}<a_{2}<\cdots<a_{s}$ unless otherwise stated. The symbol $s$ will always be reserved for the number of disjoint intervals that $E$ has. Here the indices of $[s]$ are considered cyclically, so $a_{s+1}=a_{1}$.

Our goal is to show that it is possible to find a basis that maximizes $E$ starting from some Grassmann necklace element and then applying a series of transformations to it.

Lemma 16. Let $E$ be an arbitrary subset of $[n]$. Write $E$ as in Remark 15. Let $i$ be any element of $[s]$. Then there exists a basis $B$ that maximizes $E$ and satisfies $B \cap\left(b_{i-1}, b_{i}\right]=$ $I_{a_{i}} \cap\left(b_{i-1}, b_{i}\right]$. 
Proof. Let $B$ be a basis which maximizes $E$. Pick any $e \in B \cap\left(b_{i-1}, a_{i}\right) \backslash I_{a_{i}}$. By the basis exchange axiom, there is an $e^{\prime} \in I_{a_{i}} \backslash B$ such that $(B \backslash\{e\}) \cup\left\{e^{\prime}\right\}$ is a basis; furthermore, this maximizes $E$. Set this as the new $B$, then repeat the process until the set $B \cap\left(b_{i-1}, a_{i}\right) \backslash I_{a_{i}}$ becomes empty.

Now, let $e^{\prime} \in I_{a_{i}} \cap\left[a_{i}, b_{i}\right] \backslash B$. By the dual basis exchange axiom, there is an $e \in B \backslash I_{a_{i}}$ such that $(B \backslash\{e\}) \cup\left\{e^{\prime}\right\}$ is a basis; furthermore, this maximizes $E$. Set this as the new $B$, and repeat the process until we run out of elements in $I_{a_{i}} \cap\left[a_{i}, b_{i}\right] \backslash B$.

In particular, $B$ as above will minimize $\left(b_{i-1}, a_{i}\right)$ and maximize $\left[a_{i}, b_{i}\right]$.

Example 17. To illustrate the above lemma with our running example, let $E=[1,4] \cup$ $[6,7]$. The set $B^{0}=\{1,3,6,7,10,11,14\}$ happens to be a basis which maximizes $E$. Recall that $I_{1}=\{1,3,4,5,10,11,12\}$. By exchanging to get $B^{1}:=\left(B^{0} \backslash\{14\}\right) \cup\{12\}$, we have another basis which maximizes $E$ and satisfies the condition that $B^{1} \cap(7,1)=I_{1} \cap(7,1)$. By exchanging again to get $B^{2}=\left(B^{1} \backslash\{6\}\right) \cup\{4\}$, we arrive at a final basis $B^{2}$ satisfying the condition that $B^{2} \cap[1,4]=I_{1} \cap[1,4]$.

Now we develop a method of constructing a basis that maximizes $E$, starting from some element of the Grassmann necklace. Fix some cyclically ordered elements $b, c, d \in[n]$. Notice that the number of elements a basis can have in the interval $(b, c)$ is bounded below by $\left|I_{c} \cap(b, c)\right|$ and the number of elements a basis can have in the interval $[c, d]$ is bounded above by $\left|I_{c} \cap[c, d]\right|$. We will say that a set $J$ is compatible with $I_{c}$ in $(b, d]$ if $J \cap(b, c) \supseteq I_{c} \cap(b, c)$ and $J \cap[c, d] \subseteq I_{c} \cap[c, d]$. When we are comparing such $J$ with $I_{c}$, we will call the elements of $\left(J \backslash I_{c}\right) \cap(b, c)$ as the excessive elements and the elements of $\left(I_{c} \backslash J\right) \cap[c, d]$ as the gaps.

A set $J$ mimics $I_{c}$ in $(b, d]$ if it is compatible with $I_{c}$ in $(b, d]$ and at least one of the above containments is an equality. For a set $J$ that mimics $I_{c}$ in $(b, d]$, if we have $J \cap[c, d]=I_{c} \cap[c, d]$, we say that $J$ is $\boldsymbol{g a p}$-free (with respect to $I_{c}$ in $(b, d]$ ). Otherwise we say that $J$ has gaps (with respect to $I_{c}$ in $(b, d]$ ).

Remark 18. For any cyclically ordered $a, b, c, d$, we have that $I_{a}$ is compatible with $I_{c}$ in $(b, d]$ from the sharing property, but it doesn't necessarily mimic $I_{c}$ in the same interval.

Let $J$ be a basis of $\mathcal{M}$ that is compatible to $I_{c}$ in $(b, d]$, where $b, c, d$ are cyclically ordered elements of $[n]$. Our goal is to transform $J$ into a basis that mimics $I_{c}$ in $(b, d]$. To achieve this we will replace the elements of $\left(J \backslash I_{c}\right) \cap(b, c)$ with those of $\left(I_{c} \backslash J\right) \cap[c, d]$. Let $\alpha$ be $\min \left(\left|\left(J \backslash I_{c}\right) \cap(b, c)\right|,\left|\left(I_{c} \backslash J\right) \cap[c, d]\right|\right)$. Define $J^{\prime}$ to be the set obtained from $J$ by replacing the biggest (with respect to $\left.<_{b}\right) \alpha$ elements of $\left(J \backslash I_{c}\right) \cap(b, c)$ with the smallest (again with respect to $\left.<_{b}\right) \alpha$ elements of $\left(I_{c} \backslash J\right) \cap[c, d]$. We will say that $J^{\prime}$ is obtained from $J$ by mimicking $\boldsymbol{I}_{\boldsymbol{c}} \boldsymbol{i n}(\boldsymbol{b}, \boldsymbol{d}]$. We will describe the process of using excessive elements of $\left(J \backslash I_{c}\right) \cap(b, c)$ being moved to fill the gaps of $\left(I_{c} \backslash J\right) \cap[c, d]$. The newly created $J^{\prime}$ mimics $I_{c}$. We say that this mimicking process has gaps or is gap-free depending on whether $J^{\prime}$ has gaps or is gap-free (with respect to $I_{c}$ in $(b, d]$ ).

Now we will use the above process multiple times, starting from a Grassmann necklace element, and produce multiple sets that will potentially be a basis that maximizes $E$ (again using Remark 15). We dedicate $J^{0}$ to stand for $I_{a_{1}}$. Recursively, $J^{t}$ is going to be obtained 
from $J^{t-1}$ by mimicking $I_{a_{t+1}}$ in $\left(b_{t}, b_{s}\right]$ for $t \in\{1, \ldots, s-1\}$ (this is possible since $J^{t-1}$ is compatible with $I_{a_{t+1}}$ in $\left.\left(b_{t}, b_{s}\right]\right)$. We call this process the $t$-th $\boldsymbol{m o r p h}$ of $J^{0}=I_{a_{1}}$. So we will say that the set $J^{t}$ is obtained from $J^{0}$ by morphing $t$ times. Similarly, we will use $J_{i}^{t}$ to denote the set obtained from $I_{a_{i}}$ by morphing $t$ times $\left(J_{i}^{t}\right.$ is obtained from $J_{i}^{t-1}$ by mimicking $I_{a_{t+i}}$ in $\left.\left(b_{t+i-1}, b_{s+i-1}\right]\right)$.

Example 19. Continuing with our running example, consider the set $E=[2,4] \cup[7,10]$. We label $a_{1}=2, b_{1}=4, a_{2}=7, b_{2}=10$. The set $J_{1}^{0}$ is defined as $I_{2}=\{2,3,4,5,10,11,12\}$. The first morph of $J_{1}^{0}$ will be mimicking $I_{7}$ in $(4,10]$. From $I_{7}=\{7,8,9,10,11,12,4\}$, we move the excessive elements $\left(J_{1}^{0} \backslash I_{7}\right) \cap(4,7)=\{5\}$ to fill the gaps of $\left(I_{7} \backslash J_{1}^{0}\right) \cap[7,10]=$ $\{7,8,9\}$. This gives us $J_{1}^{1}=\{2,3,4,7,10,11,12\}$, which has gaps (with respect to $I_{7}$ in $[7,10])$. The reader should be aware that we do not know if $J_{1}^{1}$ is actually a basis of $\mathcal{M}$ yet. Similarly, the set $J_{2}^{0}$ is defined as $I_{7}=\{7,8,9,10,11,12,4\}$. The first morph of $J_{2}^{0}$ will be mimicking $I_{2}$ in $(10,4]$. Since there are no excessive elements in $(10,2)$, we have $J_{2}^{1}=J_{2}^{0}$ in this case.

By way of the sharing property, the following analogue exists for $J_{i}^{t}$ 's.

Lemma 20 (Sharing property for the morphs). We have $J_{i}^{t} \cap\left[a_{i+1}, a_{i}\right) \subseteq J_{i+1}^{t-1} \cap\left[a_{i+1}, a_{i}\right)$.

Our ultimate goal is to show that one of the $J_{i}^{t}$ 's will maximize $E$.

Lemma 21. Fix a subset $E$ of the ground set as in Remark 15 . Fix some $1 \leqslant t \leqslant s-1$, then consider the set $J^{t}$. For each $1 \leqslant p \leqslant s$, there exists some nonnegative integer $q$ and a sequence $i_{1}<\cdots<i_{q}<i_{q+1}=p$ such that $J^{t}$ maximizes $\left[a_{1}, b_{i_{1}}\right],\left[a_{i_{1}+1}, b_{i_{2}}\right], \ldots,\left[a_{i_{q}+1}, b_{p}\right]$.

Proof. Recall that the $t$-th morph removes the excessive elements in $\left(b_{t}, a_{t+1}\right)$ and fills the gaps of $\left[a_{t+1}, b_{s}\right]$ from left to right. Consider the intervals $\left[a_{1}, b_{1}\right],\left[a_{2}, b_{2}\right], \ldots,\left[a_{p}, b_{p}\right]$ which are some of the components of $E$. We will associate a number on each interval in the following way: for each $\left[a_{x}, b_{x}\right]$, let $\gamma(x)$ denote the biggest number within $\{0, \ldots, \max (t, x-1)\}$ such that all gaps of $\left[a_{\gamma(x)+1}, b_{x}\right]$ gets filled in the $\gamma(x)$-th morph (that is, when $\left.J^{\gamma(x)} \cap\left[a_{\gamma(x)+1}, b_{x}\right]=I_{a_{\gamma(x)+1}} \cap\left[a_{\gamma(x)+1}, b_{x}\right]\right)$. Such a number is guaranteed to exist, since $J^{0}=I_{a_{1}}$. Now $J^{t}$ maximizes $\left[a_{\gamma(x)+1}, b_{x}\right]$ in $\mathcal{M}$, since the morphs after the $\gamma(x)$-th morph do not change the number of elements in that interval. Starting from $p$, take $\gamma(p), \gamma(\gamma(p)), \ldots$ until you get 0 . Delete 0 from this collection, and relabel them as $i_{1}<i_{2}<\cdots$ to get the desired result.

From the above lemma, we are guaranteed that each $J^{t}$ maximizes some set in $\left[a_{1}, b_{t}\right]$ (setting $p$ as $t$ ) which is obtained from $E \cap\left[a_{1}, b_{t}\right]$ by merging some nearby intervals and replacing them with a bigger interval (for example merging $[1,3] \cup[6,9]$ to get $[1,9]$ ). Now if $J^{t}$ was gap free (that is the morph to get $J^{t}$ from $J^{t-1}$ is gap-free) then $\left[a_{t+1}, b_{s}\right]$ is also maximized. In other words, $J^{t}$ that is gap free will maximize some set that is obtained from $E$ by merging some nearby intervals.

The remainder of this section will be dedicated to showing that there is some $i$ and $t$ such that $J_{i}^{t}$ is gap free and is a basis of the positroid. The next section will use that result to obtain our main result. 
Lemma 22. Let $b, c, d, e$ be cyclically ordered elements of the ground set $[n]$ and let $J$ be a basis of $\mathcal{M}$ that is compatible with $I_{c}$ in $(b, d]$. Define $J^{\prime}$ to be obtained from $J$ by mimicking $I_{c}$ in $(b, d]$. The following holds:

- If $J^{\prime} \in \mathcal{M}$ and $J^{\prime}$ has gaps (with respect to $I_{c}$ in $(b, d]$ ), then we have $|J \cap(z, b]| \geqslant$ $\left|I_{c} \cap(z, b]\right|$ for all $z \in(d, b]$.

- If $|J \cap(z, b]| \geqslant\left|I_{c} \cap(z, b]\right|$ for all $z \in(d, b]$, then $J^{\prime} \in \mathcal{M}$. Moreover if we have $J^{\prime} \cap[c, e) \subseteq I_{c} \cap[c, e)$, then the above inequality holding for all $z \in[e, b]$ is enough to get $J^{\prime} \in \mathcal{M}$.

Proof. From the fact that $J^{\prime} \backslash J>_{z} J \backslash J^{\prime}$ for any $z \notin(b, d]$ and using Corollary 7, it is enough to show $J^{\prime} \geqslant_{c} I_{c}$ in order to achieve $J^{\prime} \in \mathcal{M}$. Therefore $J^{\prime} \in \mathcal{M}$ is equivalent to $\left|J^{\prime} \cap[c, z]\right| \leqslant\left|I_{c} \cap[c, z]\right|$ for all $z \in[n]$. Since $J$ and $J^{\prime}$ are compatible with $I_{c}$ in $(b, d]$ the inequality automatically holds for any $z \in(b, d]$ (for any $z \in(b, e)$ as well). Hence we only need to show $\left|J^{\prime} \cap(z, c)\right| \geqslant\left|I_{c} \cap(z, c)\right|$ for all $z \in(d, b]$ (actually for any $z \in[e, b]$ ). Observe that $\left|J^{\prime} \cap(z, c)\right|=|J \cap(z, c)|-\alpha$ for $z \in(d, b]$, where $\alpha$ is the minimum of $\left|\left(I_{c} \backslash J\right) \cap[c, d]\right|$ (process is gap-free) and $\left|\left(J \backslash I_{c}\right) \cap(b, c)\right|$ (has gaps). Cleaning up the inequalities in the latter case gives us the desired results.

Using the above lemma, we will finish off this section with the following result.

Proposition 23. Let $\mathcal{M}$ be a positroid over the ground set $[n]$ and $E=\left[a_{1}, b_{1}\right] \cup \cdots\left[a_{s}, b_{s}\right]$ be a subset of the ground set. Again consider the sets of the form $J_{i}^{t}$ for $1 \leqslant t \leqslant s-1$, obtained from $I_{a_{i}}$ by morphing $t$ times with respect to $E$. Fix some $h$ with $1 \leqslant h \leqslant s-1$. If $J_{i}^{t} \in \mathcal{M}$ and has gaps for all $i \in[s]$ and $1 \leqslant t<h$, then $J_{i}^{h} \in \mathcal{M}$ for all $i \in[s]$.

Proof. First of all, we will show that $J^{1} \in \mathcal{M}$. Following the proof of Lemma 22, having $J^{1} \in \mathcal{M}$ is equivalent to $\left|J^{1} \cap\left(z, a_{2}\right)\right| \geqslant\left|I_{a_{2}} \cap\left(z, a_{2}\right)\right|$ for all $z \in\left(b_{2}, b_{1}\right]$. Since $J^{1} \cap\left[a_{1}, a_{2}\right) \supseteq$ $I_{a_{2}} \cap\left[a_{1}, a_{2}\right)$ and $J^{1} \cap\left(b_{2}, a_{1}\right) \subseteq I_{a_{2}} \cap\left(b_{2}, a_{1}\right)$, it is enough to show that $\left|J^{1} \cap\left(b_{2}, a_{2}\right)\right| \geqslant$ $\left|I_{a_{2}} \cap\left(b_{2}, a_{2}\right)\right|$ in order to get $J^{1} \in \mathcal{M}$. But this follows from $J^{1} \cap\left[a_{2}, b_{2}\right] \subseteq I_{a_{2}} \cap\left[a_{2}, b_{2}\right]$.

Hence we only need to consider the case when $h>1$. We will show that if $J^{1}, \ldots, J^{h-1}$, $J_{2}^{1}, \ldots, J_{2}^{h-1}$ are bases of $\mathcal{M}$ and have gaps, then $J^{h} \in \mathcal{M}$. Also notice that $J^{h} \cap$ $\left[a_{i_{h+1}}, a_{1}\right) \subseteq I_{a_{i_{h+1}}} \cap\left[a_{i_{h+1}}, a_{1}\right)$. Therefore in order to get $J^{h} \in \mathcal{M}$, Lemma 22 tells us that it is enough to show that $\left|J^{h-1} \cap\left(z, b_{h}\right]\right| \geqslant\left|I_{a_{h+1}} \cap\left(z, b_{h}\right]\right|$ for all $z \in\left[a_{1}, b_{h}\right]$.

From lemma 21, there exists a sequence $i_{1}<\cdots<i_{q}<i_{q+1}=h$ (setting $t=h-1$ and $p=h$ in the lemma) such that $J^{h-1}$ maximizes $\left[a_{1}, b_{i_{1}}\right], \ldots,\left[a_{i_{q}+1}, b_{h}\right]$ in $\mathcal{M}$. Since $I_{a_{1}}$ and $J^{h-1}$ have the same number of elements in $\left[a_{1}, b_{i_{1}}\right]$ and $I_{a_{1}} \leqslant a_{1} J^{h-1}$, we have $\left|J^{h-1} \cap\left(z, b_{i_{1}}\right]\right| \geqslant\left|I_{a_{1}} \cap\left(z, b_{i_{1}}\right]\right|$ for all $z \in\left[a_{1}, b_{i_{1}}\right]$.

Since $J^{1}, \ldots, J^{h-1}$ all have gaps, we have that $J^{h-1}$ minimizes each $\left(b_{i}, a_{i+1}\right)$ within $\left(b_{s}, b_{h}\right]$. Since $J_{2}^{1}, \ldots, J_{2}^{h-1}$ all have gaps, we have that $J_{2}^{h-2}$ minimizes each $\left(b_{i}, a_{i+1}\right)$ within $\left(b_{1}, b_{h}\right]$. This implies that within intervals of the form $\left(b_{i}, a_{i+1}\right)$ contained in $\left(b_{1}, b_{h}\right]$, the sets $J^{h-1}$ and $J_{2}^{h-2}$ are exactly the same. From the sharing property of morphs, the fact that $J^{h-1}$ maximizes $\left[a_{1}, b_{i_{1}}\right], \ldots,\left[a_{i_{q}+1}, b_{h}\right]$ implies $J_{2}^{h-2}$ also does, except potentially at $\left[a_{1}, b_{i_{1}}\right]$. Combining these facts we get that $J^{h-1} \cap\left(b_{i_{1}}, b_{h}\right]=J_{2}^{h-2} \cap\left(b_{i_{1}}, b_{h}\right]$. 
We now have all the ingredients to show that $\left|J^{h-1} \cap\left(z, b_{h}\right]\right| \geqslant\left|I_{a_{h+1}} \cap\left(z, b_{h}\right]\right|$ for all $z \in\left[a_{1}, b_{h}\right]$. Since $J_{2}^{h-1} \in \mathcal{M}$ and has gaps, Lemma 22 tells us that the same inequality replacing $J^{h-1}$ with $J_{2}^{h-2}$ is true for $z \in\left(b_{1}, b_{h}\right]$. Therefore for any $z \in\left(b_{i_{1}}, b_{h}\right]$, we have $\left|J^{h-1} \cap\left(z, b_{h}\right]\right|=\left|J_{2}^{h-2} \cap\left(z, b_{h}\right]\right| \geqslant\left|I_{a_{h+1}} \cap\left(z, b_{h}\right]\right|$. For any $z \in\left[a_{1}, b_{i_{1}}\right]$, we have $\left|J^{h-1} \cap\left(z, b_{h}\right]\right|=\left|J^{h-1} \cap\left(z, b_{i_{1}}\right]\right|+\left|J^{h-1} \cap\left(b_{i_{1}}, b_{h}\right]\right| \geqslant\left|I_{a_{1}} \cap\left(z, b_{i_{1}}\right]\right|+\left|J_{2}^{h-2} \cap\left(b_{i_{1}}, b_{h}\right]\right| \geqslant$ $\left|I_{a_{h+1}} \cap\left(z, b_{h}\right]\right|$, since we have $I_{a_{1}} \cap\left(z, b_{i_{1}}\right] \supseteq I_{a_{h+1}} \cap\left(z, b_{i_{1}}\right]$ from the sharing property.

The above proposition will be a key idea used in the proof of the main result in the next section.

\section{Rank of arbitrary sets}

Let $E$ be a subset of the ground set as in Remark 15 . We use $E_{i}$ to denote $\left[a_{i}, b_{i}\right]$. The rank of $E$ is bounded above by $\operatorname{rk}(\mathcal{M})$ minus the sum of the minimal number of elements that a basis of $\mathcal{M}$ can possibly have in each cyclic interval of the complement of $E$. So we get $\operatorname{rk}(E) \leqslant \operatorname{rk}(\mathcal{M})-\sum_{i} \operatorname{minelts}\left(b_{i}, a_{i+1}\right)$, where minelts $(b, a)$ stands for the minimal number of elements that a basis of $\mathcal{M}$ can have in the interval $(b, a)$. We call this bound the natural rank bound of $\boldsymbol{E}: \operatorname{nbd}(E):=\operatorname{rk}(\mathcal{M})-\sum_{i}\left(\operatorname{minelts}\left(b_{i}, a_{i+1}\right)\right)$. Notice that $\operatorname{minelts}(b, a)=\operatorname{rk}(\mathcal{M})-\operatorname{rk}([a, b])$.

Definition 24. Let $\Pi=T_{1} \sqcup \cdots \sqcup T_{p}$ be a partition of $[s]$ into pairwise disjoint non-empty subsets. We say that $\Pi$ is a non-crossing partition if there are no cyclically ordered $a, b, c, d$ such that $a, c \in T_{i}$ and $b, d \in T_{j}$ for some $i \neq j$. We will call the $T_{i}$ 's the blocks of the partition.

To illustrate with a simple example, $\{1,3\} \sqcup\{2\} \sqcup\{4\}$ is a non-crossing partition of [4], but $\{1,3\} \sqcup\{2,4\}$ is not. This can be easily verified by drawing the points 1 to 4 on a circle and trying to cut the circle into distinct regions corresponding to the partitions; this can only be done in the case of non-crossing partitions.

Let $\Pi$ be an arbitrary non-crossing partition of $[s]$ with $T_{1}, \ldots, T_{p}$ as its blocks. We define $\left.E\right|_{T_{i}}$ as the subset of $E$ obtained by taking only the intervals indexed by elements of $T_{i}$. For example, $\left.E\right|_{\{1,3\}}$ would stand for $E_{1} \cup E_{3}$. By submodularity of the rank function, we get another upper bound on the rank of $E: \operatorname{rk}(E) \leqslant \operatorname{rk}\left(\left.E\right|_{T_{1}}\right)+\cdots+\operatorname{rk}\left(\left.E\right|_{T_{p}}\right) \leqslant$ $\operatorname{nbd}(E, \Pi):=\operatorname{nbd}\left(\left.E\right|_{T_{1}}\right)+\cdots+\operatorname{nbd}\left(\left.E\right|_{T_{p}}\right)$. So for each non-crossing partition of $[s]$, we get an upper bound on the rank of $E$. We show that one of those bounds has to be tight in the theorem below.

Theorem 25. Let $E=\left[a_{1}, b_{1}\right] \cup \cdots \cup\left[a_{s}, b_{s}\right]$ be a disjoint union of s cyclic intervals, where $a_{1}, b_{1}, a_{2}, b_{2}, \ldots, a_{s}, b_{s}$ are cyclically ordered. We have $\operatorname{rk}(E)=\min _{\forall \Pi \in \mathrm{NC}([s])} \operatorname{nbd}(E, \Pi)$, where $\mathrm{NC}([s])$ denotes the set of all non-crossing partitions of $[s]$.

Proof. We use induction on $s$, the number of disjoint cyclic intervals of $E$. In case $s=1$, we have $\operatorname{rk}(E)=\operatorname{rk}(\mathcal{M})-\operatorname{minelts}\left(E^{c}\right)=\operatorname{nbd}(E)=\operatorname{nbd}(E,\{\{1\}\})$. Assume for the sake of induction that the claim is true for $1, \ldots, s-1$ intervals. We define $J_{i}^{t}$ recursively as 
in the previous section. From Proposition 23 (and also that $J_{i}^{1} \in \mathcal{M}$ from the proof of Proposition 23), we either have some $J_{i}^{t} \in \mathcal{M}$ that is gap-free or we have $J^{1}, \ldots, J^{s-1} \in \mathcal{M}$ all having gaps. In the latter case, since $J^{s-1}$ minimizes every interval of form $\left(b_{i}, a_{i+1}\right)$, we have $\left|J^{s-1} \cap E\right|=\operatorname{nbd}(E)=\operatorname{nbd}(E,\{\{1, \ldots, s\}\})$.

Therefore we only have to take care of the case when we have some $J_{i}^{t} \in \mathcal{M}$ that is gap-free. Pick a gap-free $J_{i}^{t}$ with minimal $t$. Without loss of generality, we will assume $i=1$. From Lemma 21, we have some sequence $i_{1}<\cdots<i_{q}<i_{q+1}=t$ such that $J^{t}$ maximizes $\left[a_{1}, b_{i_{1}}\right], \ldots,\left[a_{i_{q}+1}, b_{t}\right],\left[a_{t+1}, b_{s}\right]$ (the last interval is maximized due to $J^{t}$ being gap-free). We will use $F_{1}, \ldots, F_{q+2}$ to denote these intervals. For each $1 \leqslant j \leqslant q+2$, let $K_{j}$ be a basis that maximizes $F_{j} \cap E$. Pick $K_{j}$ using Lemma 16 so that it minimizes the complement of $F_{j}$ in $[n]$ as well. Since $\left|K_{j} \cap F_{j}\right|=\left|J^{t} \cap F_{j}\right|$, using Proposition 4 we can replace $J^{t} \cap F_{j}$ with $K_{j} \cap F_{j}$ in $J^{t}$ for each $j$ to obtain a new basis $B$. By induction hypothesis, for each $j$, we have $\left|B \cap F_{j} \cap E\right|=\operatorname{rk}\left(F_{j} \cap E\right)=\operatorname{nbd}\left(F_{j} \cap E, \Pi_{j}\right)$ for some non-crossing partition $\Pi_{j}$. Letting $\Pi$ be a non-crossing partition obtained by collecting (and relabeling so the elements match the indices of intervals in $F_{j} \cap E$ ) all blocks of $\Pi_{j}$ 's, we get $|B \cap E|=\operatorname{rk}(E)=\operatorname{nbd}(E, \Pi)$.

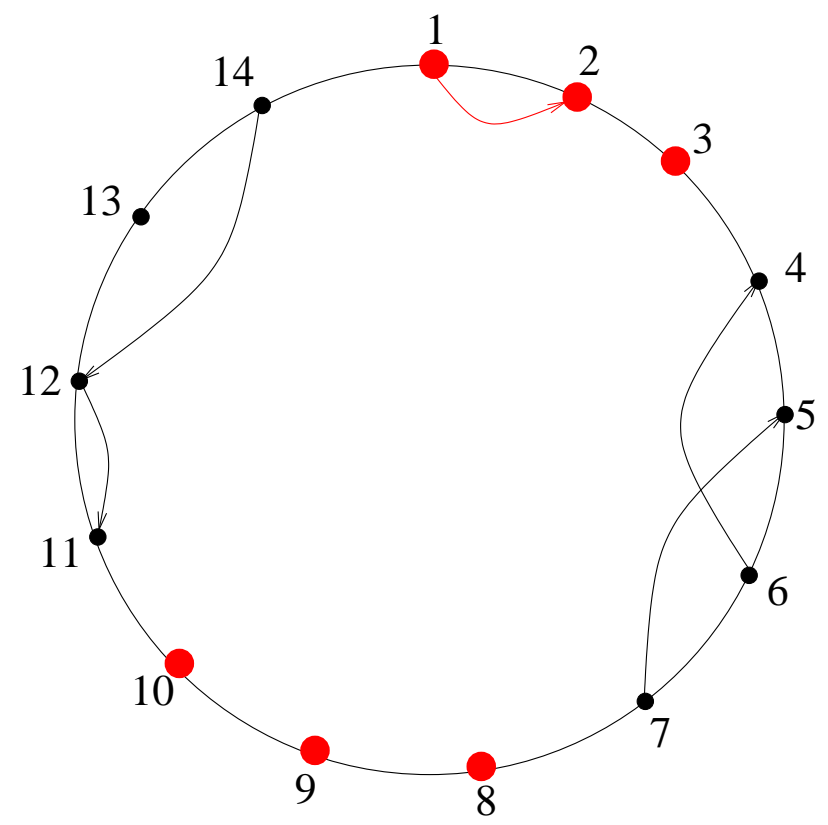

Figure 2: Information needed to compute the rank of $[1,3] \cup[8,10]$.

Example 26. Continuing with our running example, take a look at Figure 2. The rank of $E=[1,3] \cup[8,10]$ is bounded above by $\operatorname{nbd}(E,\{\{1\},\{2\}\})$ and $\operatorname{nbd}(E,\{\{1,2\}\})$. We get $\operatorname{nbd}(E,\{\{1\},\{2\}\})=\operatorname{rk}([1,3])+\operatorname{rk}([8,10])=2+3=5$, since rank of an interval $[a, b]$ is given by $|[a, b]|$ minus the number of intervals of form $\left[\pi^{-1}(x), x\right]$ contained in $[a, b]$ (from $I_{a}$ being given by $a$-exceedances, and $\left.\operatorname{rk}([a, b])=\left|I_{a} \cap[a, b]\right|\right)$. We also have 
$\operatorname{nbd}(E,\{\{1,2\}\})=\operatorname{rk}(\mathcal{M})-\operatorname{minelts}((3,8))-\operatorname{minelts}((10,1))=7-2-2=3$, since minelts $((b, a))$ is given by the number of intervals of form $\left[x, \pi^{-1}(x)\right]$ contained in $(b, a)$. Hence the above theorem tells us that $\operatorname{rk}(E)=3$.

\section{Application}

Let $\mathcal{M}$ be a positroid and let $E$ be an arbitrary subset of the ground set $[n]$. In this section, we will show how to use Theorem 25 to obtain the rank of $E$. We will call an interval of form $[x, \pi(x)]$ a $\boldsymbol{C W}$-arrow, and an interval of form $\left[x, \pi^{-1}(x)\right]$ a $\boldsymbol{C} \boldsymbol{C W}$ arrow (each standing for clockwise and counterclockwise). Given a cyclic interval $T$, we use $\mathrm{cw}(T)$ to denote the number of $\mathrm{CW}$-arrows contained in $T$. Similarly, we will use $\operatorname{ccw}(T)$ for the number of CCW-arrows contained in $T$. These numbers can easily be read from the associated decorated permutation of $\mathcal{M}$.

Recall that $\operatorname{nbd}\left(\left[a_{1}, b_{1}\right] \cup \cdots \cup\left[a_{s}, b_{s}\right]\right)=\operatorname{rk}(\mathcal{M})-\sum_{i}\left(\operatorname{minelts}\left(b_{i}, a_{i+1}\right)\right)$, where we denote by minelts $\left(b_{i}, a_{i+1}\right)$ the minimal number of elements a basis can have in the interval $\left(b_{i}, a_{i+1}\right)$. This implies $\left|I_{a+1} \cap\left(b_{i}, a_{i+1}\right)\right|$, and hence minelts $\left(b_{i}, a_{i+1}\right)=\operatorname{ccw}\left(\left(b_{i}, a_{i+1}\right)\right)$. This means we can interpret $\operatorname{nbd}(E)$ as $\operatorname{rk}(\mathcal{M})$ minus the total number of CCW-arrows contained in the complement of $E$. In the special case when $E$ is a cyclic interval, $\operatorname{nbd}(E)$ is given by $|E|$ minus the number of $\mathrm{CW}$-arrows contained in $E$.

Therefore for any $E$, we can obtain $\operatorname{nbd}(E, \Pi)$ by counting $\mathrm{CW}$-arrows and CCWarrows. If $E$ is the disjoint union of $s$ cyclic intervals, we first write all possible noncrossing partitions of $[s]$. Each one of them gives a sum of $\operatorname{nbd}\left(E^{\prime}\right)^{\prime}$ s where $E^{\prime}$ obtained from $E$ by taking some of the $s$ cyclic intervals of $E$, and we compute them by counting the CCW-arrows (or CW-arrows for intervals) of the decorated permutation.

Continuing with our running example (the positroid associated with Figure 1), let us try to compute the rank for $E=[1,2] \cup[7,10] \cup[13,13]$. We have 3 disjoint intervals, so the upper bounds of $\operatorname{rk}(E)$ will be coming from the non-crossing partitions of $\{1,2,3\}$. The following are the upper bounds for $\operatorname{rk}(E)$ we get:

- $\operatorname{nbd}(E,\{\{1,2,3\}\})=\operatorname{nbd}(E)=\operatorname{rk}(\mathcal{M})-\operatorname{ccw}((2,7))-\operatorname{ccw}((10,13))-\operatorname{ccw}((13,13))$ $=7-1-1-0=5$.

- $\operatorname{nbd}(E,\{\{1\},\{2,3\}\})=\operatorname{nbd}\left(E_{1}\right)+\operatorname{nbd}\left(E_{2} \cup E_{3}\right)=|[1,2]|-\operatorname{cw}([1,2])+\operatorname{rk}(\mathcal{M})-$ $\operatorname{ccw}((10,13))-\operatorname{ccw}((13,7))=1+7-1-1=6$.

- $\operatorname{nbd}(E,\{\{1,2\},\{3\}\})=\operatorname{nbd}\left(E_{1} \cup E_{2}\right)+\operatorname{nbd}\left(E_{3}\right)=\operatorname{rk}(\mathcal{M})-\operatorname{ccw}((2,7))-$ $\operatorname{ccw}((10,1))+|[13,13]|-\operatorname{cw}((13,13))=7-1-2+1-0=5$.

- $\operatorname{nbd}(E,\{\{1,3\},\{2\}\})=\operatorname{nbd}\left(E_{1} \cup E_{3}\right)+\operatorname{nbd}\left(E_{2}\right)=\operatorname{rk}(\mathcal{M})-\operatorname{ccw}((2,13))-$ $\operatorname{ccw}((13,1))+|[7,10]|-\operatorname{cw}([7,10])=7-5-0+4-0=6$.

- $\operatorname{nbd}(E,\{\{1\},\{2\},\{3\}\})=\operatorname{nbd}\left(E_{1}\right)+\operatorname{nbd}\left(E_{2}\right)+\operatorname{nbd}\left(E_{3}\right)=|[1,2]|-\operatorname{cw}([1,2])+$ $|[7,10]|-\mathrm{cw}([7,10])+|[13,13]|-\mathrm{cw}([13,13])=2-1+4-0+1-0=6$. 
Theorem 25 tells us that $\operatorname{rk}(E)=5$.

Using Theorem 25, in [11] it is shown that the facets of the matroid polytope are given by cyclic intervals whose complement is covered by CCW-arrows. It is also shown that the facets of the independendent set polytope of a positroid are given by sets whose complement is again covered by CCW-arrows. In [3], the condition for an arbitrary subset of the ground set being a flat of the positroid will be given in terms of the decorated permutation, again using Theorem 25 .

\section{References}

[1] F. Ardila, F. Rincon, and L. Williams. Positroids and non-crossing partitions. Transactions of the American Mathematical Society, 368(1):337-363, (2016).

[2] N. Arkani-Hamed, J. L. Bourjaily, F. Cachazo, A. B. Goncharov, A. Postnikov, and J. Trnka. Scattering Amplitudes and the Positive Grassmannian. arXiv:1212.5605

[3] B. Chen, A. Lu, S. Oh, and C. Zhou. Flats of a positroid. in preparation.

[4] S. Fomin and A. Zelevinsky. Total positivity: Tests and parametrizations. Mathematical Intelligencer, 22(1):23-33, (2000).

[5] D. Gale. Optimal assignments in an ordered set: An application of matroid theory. Journal of Combinatorial Theory, 4(2):176-180, (1968).

[6] A. Knutson. Schubert Calculus and Shifting of Interval Positroid Varieties. arXiv: 1408.1261

[7] Y. Kodama and L. Williams. Kp solitons and total positivity for the grassmannian. Inventiones mathematicae, 198(3):637-699, (2014).

[8] T. Lam. Electroid varieties and a compactification of the space of electrical networks. Advances in Mathematics, 338:549 - 600, (2018).

[9] T. Lam and A. Postnikov. Polypositroids. in preparation.

[10] S. Oh. Positroids and schubert matroids. Journal of Combinatorial Theory, Series A, 118(8):2426-2435, (2011).

[11] S. Oh and D. Xiang. The facets of the matroid polytope and the independent set polytope of a positroid. arXiv:1701.08483

[12] J. Oxley. Matroid Theory. Oxford University Press, 2011.

[13] A. Postnikov. Total positivity, Grassmannians, and Networks. arXiv:0609764 